\title{
3D assessment of an underground mine pillar by combination of photogrammetric and geoelectric methods
}

\author{
Yannick Fargier ${ }^{1}$, Raphaël Antoine ${ }^{2}$, Ludovic Dore ${ }^{1}$, Sérgio Palma Lopes ${ }^{3}$, and Cyrille Fauchard ${ }^{2}$
}

\begin{abstract}
The monitoring of underground cavities plays a key role in risk management policies. Mine and underground quarry stakeholders require relevant methodologies and practices to define and assess hazards associated with these structures. To monitor these structures, geophysical methods may offer an interesting compromise among operating cost, invasiveness, and risk assessment reliability. The use of conventional 3D-electric resistivity imaging (ERI) software validated on relatively flat media is not sufficient to efficiently assess complex 3D geometries such as underground mine pillars. We have developed a new approach to evaluate pillar condition by means of a sequential use of two techniques. First, the
\end{abstract}

photogrammetric method yields a detailed 3D model of the pillar geometry from a set of pictures. Second, 3D-ERI is performed based on this suitable geometry. The methodology is tested on a synthetic model to evaluate the effect of various geometry resolutions on the inversion. We also evaluated the combination of the effect of measurement and geometry error. We performed a quasi 3D-ERI survey (three parallel electrode lines) on a real limestone mine pillar to determine the benefits and limitations of the combined procedure. First results revealed the capacity of the photogrammetric methods to obtain a high-precision geometry and its key role during the inversion process. Second results of the real case study revealed that a highly accurate geometry is required to detect accurately conductive anomalies in a complex 3D context.

\section{INTRODUCTION}

Degradation of underground quarries can lead to collapses, which can directly impact buildings and civil engineering structures on the surface and, even worse, dangerously threaten people's safety (Bell et al., 1992; Al Heib et al., 2014). In this context, the monitoring of abandoned quarries is of major importance.

Stability assessment methods of underground chalk mines are mainly based either on structural numerical modeling or on geometric criteria (i.e., tributary area method) (Brady and Brown, 2013). Both are combined with rock physical properties measured in the vicinity of the area studied. Nevertheless, these methods are based on the assumption that the internal properties of pillars are quite homogeneous (Ferrero et al., 2010), and consequently, they are likely to underestimate the associated risk of collapse.

A complementary approach consists of using geophysical methods to overcome this limitation. To this end, Dérobert and Abraham (2000) use seismic and ground-penetrating radar methods to assess pillar conditions in a gypsum quarry. Results showed that both methods were able to detect internal cracks in the medium.

Electric resistivity imaging (ERI) is a widely used method exhibiting two main benefits when applied to mine pillar assessment. The first is to provide qualitative detection and monitoring of anomalies within the pillar (e.g., cracks or heterogeneous zones [Jones et al., 2014; Li et al., 2015]). The second is to provide a quantitative description of the electric resistivity distribution within the pillar. ERI is considered to be a relevant tool to assess the internal distribution of water content in geologic (Brunet et al., 2010) or civil engineering (Rings et al., 2008) contexts. This quantitative description can be interpreted with empirical laws to link the variation in chalk mechanical properties with water content distribution (Gombert et al., 2013).

However, conventional 2D and 3D imaging techniques are mainly applied to flat surface surveys (Dahlin, 1996) or to surfaces with moderate topography (Fox et al., 1980; Günther et al., 2006; Erdoğan et al., 2008; Demirci et al., 2012; Fargier et al., 2014). Interesting

Manuscript received by the Editor 26 May 2016; revised manuscript received 23 January 2017; published online 9 May 2017.

${ }^{1}$ Cerema, Direction Territoriale Normandie Centre, Blois, France. E-mail: yannick.fargier@cerema.fr; ludovic.dore@cerema.fr.

${ }^{2}$ Cerema, Direction Territoriale Normandie Centre, Rouen, France. E-mail: raphael.antoine@cerema.fr; cyrille.fauchard@cerema.fr.

${ }^{3}$ LUNAM Université, IFSTTAR, Department GERS, Nantes, France. E-mail: sergio.palma.lopes@ifsttar.fr.

(C) 2017 Society of Exploration Geophysicists. All rights reserved. 
results can be obtained using a 3D forward model (using a complex geometry) and performing a 2D inversion (Lesparre et al., 2014). A 3D acquisition survey (multiple electrode lines) on a complex 3D medium (which is the case of a pillar) requires a full account of the geometry in the 3D inversion process (Marescot et al., 2008). Indeed, studies show that it is of prime importance to use a model closest to reality, including the lowest electrode position error (Oldenborger et al., 2005) and relevant insulating boundary conditions (Audebert et al., 2014).

Laser and photogrammetric techniques can be used to obtain a very accurate description of the external geometry of a complex medium (Chandler et al., 2005; Abellán et al., 2014). The latter often provides a cost-effective solution for building a digital terrain model (DTM) (Bretar et al., 2013). Recently, techniques based on close-range stereophotogrammetry have proven effective in generating accurate 3D models of civil engineering structures for a better interpretation of geophysical measurements (Fauchard et al., 2013). However, this method has never been fully sequentially exploited with the 3D-ERI method to improve the inversion process.

The purpose of this work is to show that photogrammetry and ERI tools can be combined to efficiently assess a complex 3D structure. First, this paper presents the general methodology with the basic principles of photogrammetry and ERI. Second, some numerical tests are performed to highlight the key role of the resolution of the $3 \mathrm{D}$ surface geometry on the inversion result. Finally, the methodology is applied to image electric resistivity distribution within a real underground pillar in a chalk mine.

\section{MATERIALS AND METHODS}

\section{Methodology}

Major ERI software optimized for the assessment of flat or moderately flat media is unsuitable for assessing mine pillars with complex geometry. We propose to sequentially combine a photogrammetric method and a flexible 3D inversion approach (for generating the model) to address this issue. Figure 1 presents a simplified diagram

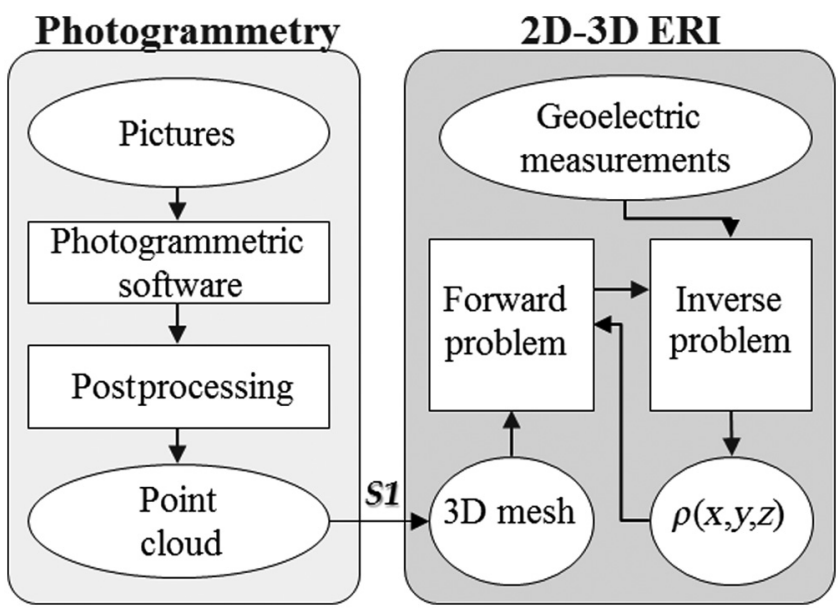

Figure 1. Methodology sequentially coupling ERI and the photogrammetric method to assess a medium with complex geometry. The rectangular elements represent software and methods. The ellipsoidal elements represent input and output data, $\rho(x, y, z)$ is the electric resistivity distribution, and $S 1$ is a denoising postprocessing stage of the raw output point cloud. of the assessment methodology coupling photogrammetric and ERI techniques. The main idea is that photogrammetry can supply an accurate geometry used as an input model for the ERI inversion process. For this study, the cost-effectiveness of photogrammetry and its flexibility led us to use this method, rather than the laser scanning technique.

\section{Photogrammetry basic principles}

Photogrammetry allows the reconstruction of high-resolution 3D point cloud models from images acquired from different locations (Chandler et al., 2005). This technique exploits the difference in the observation of one object viewed from two viewpoints, also known as the parallax principle. The mimic of this perspective shift with a set of photographs is the basic acquisition protocol used for 3D reconstruction. The rapid evolution of digital cameras and computing power has dramatically expanded the variety of potential applications for this method, while simultaneously decreasing the costs of acquisition, processing, and analysis. Photogrammetry is widely used in engineering geology applications for (1) reconstructing high-resolution 3D objects and (2) monitoring the evolution of surfaces. In the framework of this research study, the authors use the APERO/MICMAC photogrammetric software suite developed by the Institut National de l'Information Géographique et Forestière since 2007 (Pierrot-Deseilligny and Clery, 2011). MicMac software follows a classic process consisting of three successive main steps to obtain a point cloud:

1) The first step is to compute tie points between images using the scale invariant feature transform (SIFT) algorithm (Vedaldi and Fulkerson, 2010). The picture data set is then organized following the number of tie points between photos.

2) The second step of the process computes the orientations of camera for every shot and gives an initial solution minimizing a cost function. The latter is composed of a correlation cost function and a smoothness cost function (Pierrot-Deseilligny and Clery, 2011). A master image is selected that arbitrarily gives the orientation and origin of the coordinates. Next, images are selected computing the relative orientation from the tie points. At this stage, ground control points (GCP) can be used to calibrate the 3D model and to obtain an absolute orientation. A self-calibration method is used during the bundle adjustment to take the lens distortion into account.

3) The last step produces a dense $3 \mathrm{D}$ point cloud using a multiscale method (Pierrot-Deseilligny and Clery, 2011). The process uses the previous orientations of cameras and starts with a rough approximation of the 3D scene and low-resolution images. Then, the resolution of $3 \mathrm{D}$ scene is iteratively improved by a dense matching of the images at higher resolution. The dense matching is based on a pixel-based or window-based normalized crosscorrelation of images.

More details concerning the mathematical principle, the acquisition process, and the applications of the technique can be found in the literature (Pierrot-Deseilligny and Clery, 2012; Bretar et al., 2013).

\section{Geoelectric imaging principles}

The ERI method makes it possible to image the electric resistivity distribution within a medium by measuring voltage drops on its surface or boreholes with electrodes (Dahlin, 1996). 
The geoelectric inversion code used in this paper is developed with the MATLAB and Comsol Multiphysics software (Multiphysics, 2008). The former is used to solve the inverse problem and manage the latter, which solves the forward problem. The latter is a finite-element method that solves the Poisson's equation (equation 1) for each electrode as a source electrode (s) with a weak formulation approach (Günther et al., 2006):

$$
\nabla \cdot(\sigma \nabla V)=-I\left(\delta\left(r-r_{s}\right)\right)
$$

where $V$ is the electric potential $(V)$ calculated at each node of the finite-element mesh and $\sigma$ is the electric conductivities at each nodes of the mesh $(\mathrm{S} / \mathrm{m})$. The right-hand part of the equation represents the source term, with $I$ being the current intensity $(A)$ injected in the electrode located at $r_{s}(\mathrm{~m})$. Finally, Neumann and Dirichlet boundary conditions are applied to take into account insulating boundaries and ground conditions, respectively.

The inversion procedure is based on a Gauss-Newton algorithm with an Occam-type regularization (deGroot-Hedlin and Constable, 1990). This procedure is used iteratively to find a better model $\mathrm{m}^{k+1}$ by updating a model $m^{k}$ at a previous iteration $k$ (Sasaki, 1994):

$$
m^{k+1}=m^{k}+\tau \Delta m
$$

where $\tau$ is a scalar used to ensure the convergence of the algorithm and to limit the overshooting issue. It is computed with the threepoint parabola method (Günther et al., 2006). To compute the updating parameter vector $\Delta \mathbf{m}$, we solve the normal inversion equation (equation 3) (Sasaki, 1994) with a QR decomposition solver:

$$
\left[\begin{array}{c}
\mathbf{W}_{d} \mathbf{S} \\
\sqrt{\lambda} \mathbf{L}
\end{array}\right] \Delta \mathbf{m}=\left[\begin{array}{c}
\mathbf{W}_{d} \Delta \mathbf{r} \\
0
\end{array}\right],
$$

where $\mathbf{W}_{d}$ is the data-weighting matrix and $\Delta \mathbf{r}$ is the data misfit between the measured and computed data. We use an adjoint-state method to compute the sensitivity matrix (Park and Van, 1991). Regularization of the inverse problem is ensured by a conventional model smoothing matrix $\mathbf{L}$ corresponding to a second derivative of the model (deGroot-Hedlin and Constable, 1990; Sasaki, 1994). The damping factor $\lambda$ is given an initial value of one, and it is then divided by two before each subsequent iteration (Loke and Barker, 1996).

To improve the interpretation of a numerical study, a similarity index $S_{i}$ between the inversion result and the true model is proposed (equation 4). The aim of this criterion is to give an error associated with a reconstruction of a known synthetic medium. An index equal to zero means a perfect reconstruction and one means an average reconstruction error of $100 \%$ :

$$
S_{i}=\sqrt{\frac{1}{V_{i}} \int^{V_{i}}\left(\frac{\rho_{\text {inv }}-\rho_{\text {true }}}{\rho_{\text {true }}}\right)^{2} d V_{i}},
$$

where $V_{i}$ is the examined volume of interest, $\rho_{\text {inv }}$ is the inverted resistivity distribution, and $\rho_{\text {true }}$ is the real resistivity distribution in the volume.

\section{CASE STUDY}

\section{Site test description}

A real quarry pillar is examined in the following sections (synthetic and real case studies) to test the methodology. La Glacière is an ancient underground limestone quarry located in France near Vendôme (Figure 2a). The quarry covers approximately $6000 \mathrm{~m}^{2}$ and was exploited from the seventieth century until the twentieth century (Figure 2b). Because the quarry stopped being exploited, damage has occurred, such as cracking and local collapsing of the roof and pillars. Some of these cracks are linked with the local geologic faults oriented northwest-southeast. The limestone is a Cretaceous chalk (Senonian) containing flints and fossils (Figure 2a, in green). This chalk is covered by a clayey/sandy formation. Its thickness ranges between 2.5 and $13.5 \mathrm{~m}$. The total thickness of the sedimentary horizon of land above the pillar is $20 \mathrm{~m}$. In the examined area, regular pillars were built. The perimeter of the examined pillar is approximately $25 \mathrm{~m}$, and its mean height is $3 \mathrm{~m}$. It is located approximately $50 \mathrm{~m}$ away from the quarry entrance. One of the cracks linked with the geologic fault can be seen around the pillar.

Regarding a possible thermal effect on resistivity (Hayley et al., 2010), preliminary thermal infrared imaging camera observations showed that the temperature is clearly homogeneous over the whole pillar surface. Given its location in the quarry, the temperature within the pillar is considered to be homogeneous.

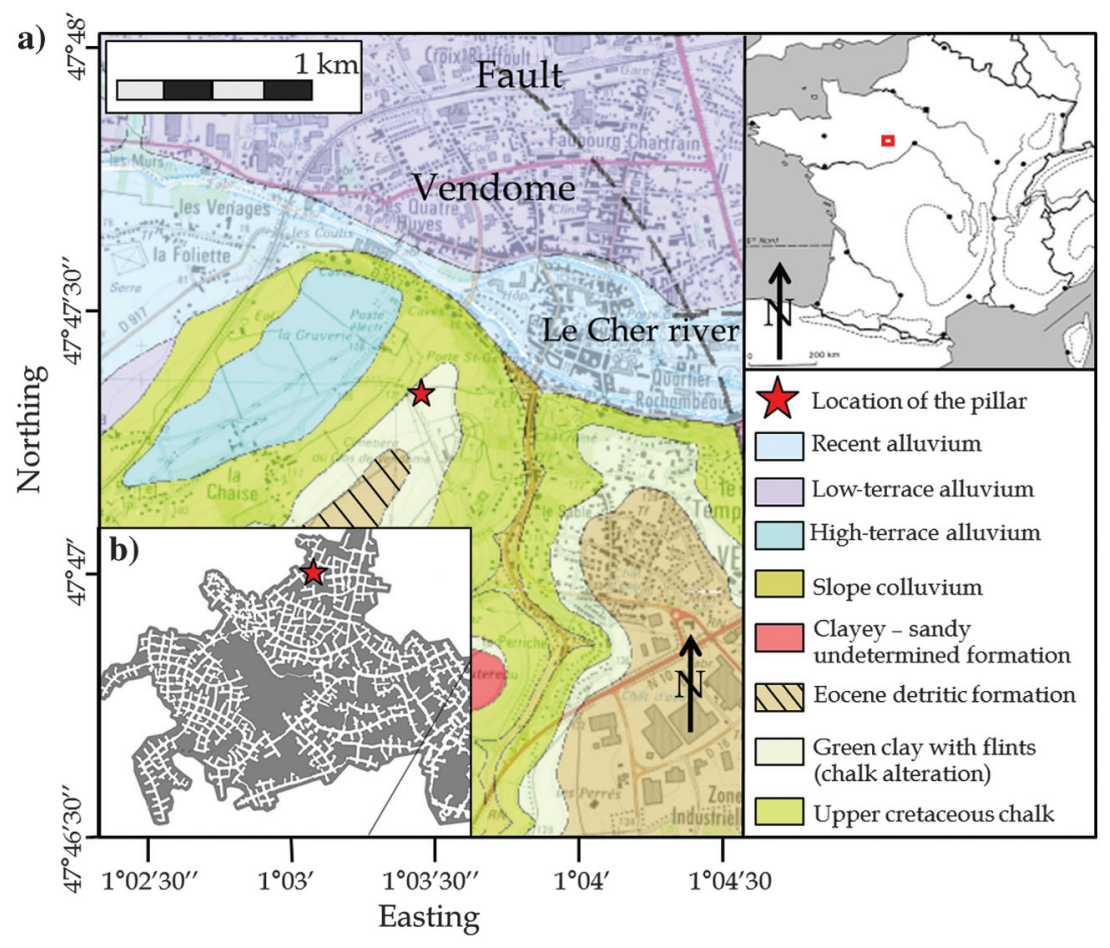

Figure 2. (a) Geologic map of the Vendôme area and (b) map of the quarry. The red star indicates the location of the pillar. Coordinate system: WGS84. 


\section{Photogrammetric survey}

To produce the photogrammetric surface model, a set of approximately 100 pictures obtained at different angles and distances was taken on the quarry pillar (see Figure 3). For this survey, we followed a linear acquisition path to get the entire data set with an average overlap of $80 \%$ between two successive photographs (Pierrot-Deseilligny and Clery, 2012) (see Figure 3). During the acquisition (approximately $1 \mathrm{~h}$ ), great attention was paid to the four corners of the pillar: More images were acquired for an optimal link between each side of the pillar.

The camera used is a Panasonic GF1 (the focal length is $28 \mathrm{~mm}$, and the sensor size is $18 \times 13.5 \mathrm{~mm}$ ), creating 12 megapixel pictures. Due to a lack of luminosity in underground context, we used six spots (500 W each) to homogenize the light on the pillar. The difficulty to homogenize the illumination of the pillar appears to be a major cause of noise in the colored point cloud (Figure 3).

We used a set of 192 GCPs corresponding to the location of the electrodes. The GCPs coordinates $(x, y, z)$ were obtained with a laser apparatus first to calibrate the photogrammetric model and second to compare the photogrammetric and laser DTM. Considering the accuracy of this method, the positioning errors are assumed to be negligible. These GCPs are included after the bundler adjustment by giving the pixel location $(x, y)$ of each GCP on 72 photographs out of the complete set of pictures. After the MICMAC computation, the final point cloud is exported to carry out a comparison between

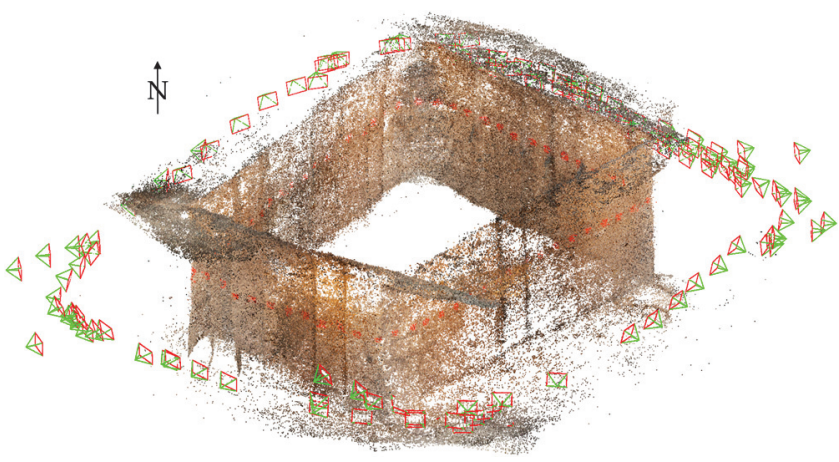

Figure 3. The 3D view of the resulting colored point cloud. The red and green tetrahedrons represent the locations and orientations of the camera.

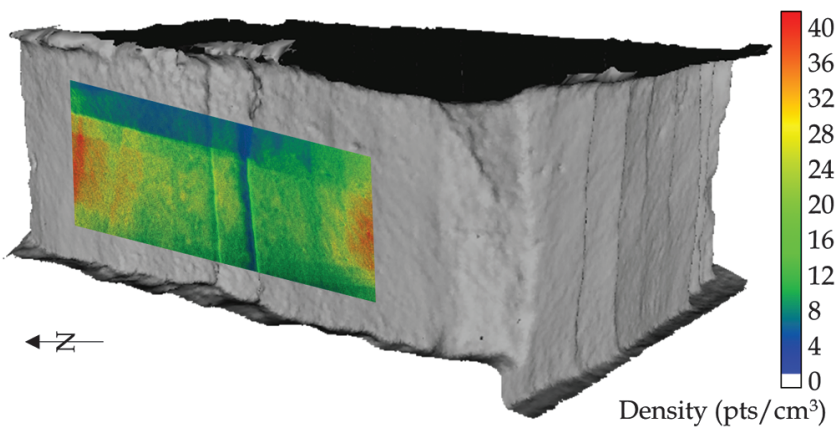

Figure 4. Photogrammetric model obtained after removing outliers and applying the denoising procedure. The superimposed figure in color shows the point density of an area of the photogrammetric model. laser and photogrammetric results. The study shows a root-meansquare (rms) error of the photogrammetric point cloud less than $2 \mathrm{~cm}( \pm 0.4 \mathrm{~cm})$ in all three directions.

Figure 4 shows the result of the photogrammetric computation after removing outliers and applying a denoising procedure. The former procedure consisted of manually removing points that fall outside of the region of interest. The denoising procedure is a statistical outlier removal (SOR) filter computing the mean distance of each point to its neighbors and rejecting points that are farther than this average distance plus a standard deviation (Point Cloud Library [PCL]). We use six neighbors for the mean distance estimation and a standard deviation of one. The average point density on each face of the pillar is 23 points $/ \mathrm{cm}^{2}$. It can be noted that the lower volume point density near the cutting faces of the pillar is due to blind spots. The resulting photogrammetric model contains approximately 10 million points.

\section{Geoelectric survey}

We performed an ERI survey on the pillar. It consists of three electrode belts around the pillar located approximately 1.0, 1.5, and $2.0 \mathrm{~m}$ high (entitled H1, H2, and H3, respectively; Figure 5b). Each belt is composed of 64 electrodes with a $37 \mathrm{~cm}$ interelectrode spacing. The total perimeter is approximately $24 \mathrm{~m}$.

For each electrode belt, a specific Wenner-Schlumberger protocol (for circular tomography [Lesparre et al., 2014]) was performed with the ABEM Terrameter LS producing three sets of 1224 quadrupole measurements $(a=1-8$ and $n=1-4)$. Protocol does not contain crossline measurements (between belts, [Cho and Yeom, 2007]). All of the measurements present an excellent quality factor (mean $=0.08 \%$, Std $=0.09$ ), and no reciprocal measurements were carried out. The electrodes are $8 \mathrm{~cm}$ length stainless steel screws embedded to a depth of $4 \mathrm{~cm}$ in the chalk. This technical solution represents the best trade-off in terms of cost and contact resistance (the measured average contact resistance is $750 \Omega$ ). Three electrodes presented a lower contact resistance (approximately $50 \Omega$ ).

\section{Model construction}

To be successfully inverted, the model (geometry and meshing) needs to fit the actual medium and physics (electric current diffusion) as accurately as possible. However, the required level of accuracy decreases proportionally to the distance from the electrodes. Consequently, it is necessary to integrate great precision in the electrode locations (Oldenborger et al., 2005) and surface topography nearby. Conversely, a lower accuracy is needed for the remainder of the model. We construct a model composed of three parts (Figure $5 \mathrm{a}-5 \mathrm{c})$. First, a $20 \mathrm{~m}$ high layer of land above the quarry is generated to let the current circulate above the "room" and the pillar. Second, the pillar is surrounded by air (Figure 5b). Third, a hemisphere of earth material (radius: $500 \mathrm{~m}$ ) is generated below the pillar to let the current circulate below the room within an "infinite" medium (Marescot et al., 2008). As a consequence, the only part of the true geometry that is neglected consists of the other pillars. Given their distance to the electrodes, we assume that they have no significant influence on the data.

Concerning the generation of the pillar, the output model of the photogrammetric survey was used. However, even after a denoising postprocessing stage (CloudCompare, Figure 4), the model was too dense and noisy to be directly used for our applications: 3D 
geoelectric modeling. Consequently, to overcome the above limitation and supply a suitable model, we chose to construct it by extrusion and interpolation of horizontal slices extracted at several heights of the previously presented photogrammetric model. More precisely, some extrusion and surface interpolation operations (loft NURBS: nonuniform rational basis splines) between the slices were performed to generate the complete $3 \mathrm{D}$ geometry model (Figure $5 \mathrm{~b}$ ). Extrusions of slices were made near the location of electrodes $\left(\mathrm{E}_{1}\right.$, $E_{2}$, and $E_{3}$ ), whereas lofts were made in between the above-mentioned zones $\left(\mathrm{L}_{1}\right.$ and $\left.\mathrm{L}_{2}\right)$ (Figure $5 \mathrm{~b}$ ). Each loft is computed with eight guided curves and four intermediate layers to avoid self-intersections. The choice to use horizontal slices comes from the need to obtain a greater horizontal accuracy of the surface geometry. Indeed, fewer surface geometry variations are expected in the vertical axis and the acquisition (no interbelt measurement) induces a maximum sensitivity between electrodes of the same belt, not between them.

After this simplification, the boundaries of the model are extended to fit the Dirichlet criteria (Figure 5a and 5c). The mesh model is shown in Figure 5a, in which the pillar is centered and surrounded by one hemisphere (beside) and a plate simulating the land above. Insulation conditions are imposed at the boundaries between the lower hemisphere and the land, at the surface of the pillar and at the top of the model (air/media boundaries, Figure $5 \mathrm{c}$ ).

\section{Numerical test}

The aim of this section is to present a numerical study showing how inversion results depend on the resolution of the 3D geometric surface model. A first model, considered as the "true model" and based on slices in full resolution (approximately 40 points between each electrode), is created according to the aforementioned methodology (see Figure 6a). Then, six distinct models of various resolutions are created (M1-M6).

A decreasing resolution is applied from the first (M1) to the sixth model (M6). For M1, 40 points (1 $\mathrm{cm}$ spacing) are used between each pair of electrodes to construct the various slices of the model (the same number of points as compared with the true model). This number is decreased to 10 points for M2 (4 cm spacing), five points for M3 (7 cm spacing), two points for M4 (13 cm spacing), one point for M5 (20 cm spacing), and zero point for M6 (40 cm spacing) slightly modifying the contour of the geometry (Figure $6 \mathrm{~b}$ for M1, M3, and M6). Consequently, for M6, the contours are only based on the "true" electrode positions (see Figure 6a). The enlargement (Figure 6b) shows the contour interpolation for M1, M3, and M6. Figure 6c-6e presents for models M1, M3, and M6 a 2D slice of the $3 \mathrm{D}$ forward mesh, and an enlargement of this mesh and the $3 \mathrm{D}$ inversion cells, respectively. The two enlargements are proposed in a particular area of interest showing the impact of a lower geometry resolution (near the most angular working face, Figure $6 \mathrm{~b}$ and 6d). For each model (M1-M6), the inversion cells are composed of four elements (Figure 6e) between each pair of electrodes (explaining a quite constant number of inversion cells and forward meshes). The difference between these models comes from the capacity of the inversion cells fitting the various accuracies of the surface geometry.

Three cylindrical anomalies (vertically oriented) are positioned in the true model of the pillar. The resistivity of the anomalies and the surrounding pillar body are 150 and $250 \Omega \mathrm{m}$, respectively (Figure 7). Anomaly A1 is located near an angular working face of the pillar, whereas A3 is close to a flat side of the pillar. To study the effect of surface geometry errors on inversion results far from the contour, a larger anomaly A2 is generated in the center of the model. A Wenner-Schlumberger protocol is then simulated for each electrode belt to collect the "measured" data detailed in the subsection "Geoelectric survey."

Figure 8 summarizes the inversion results of each model at the seventh iteration of inversion. These results are presented from slices extracted at three heights corresponding to the electrode belts

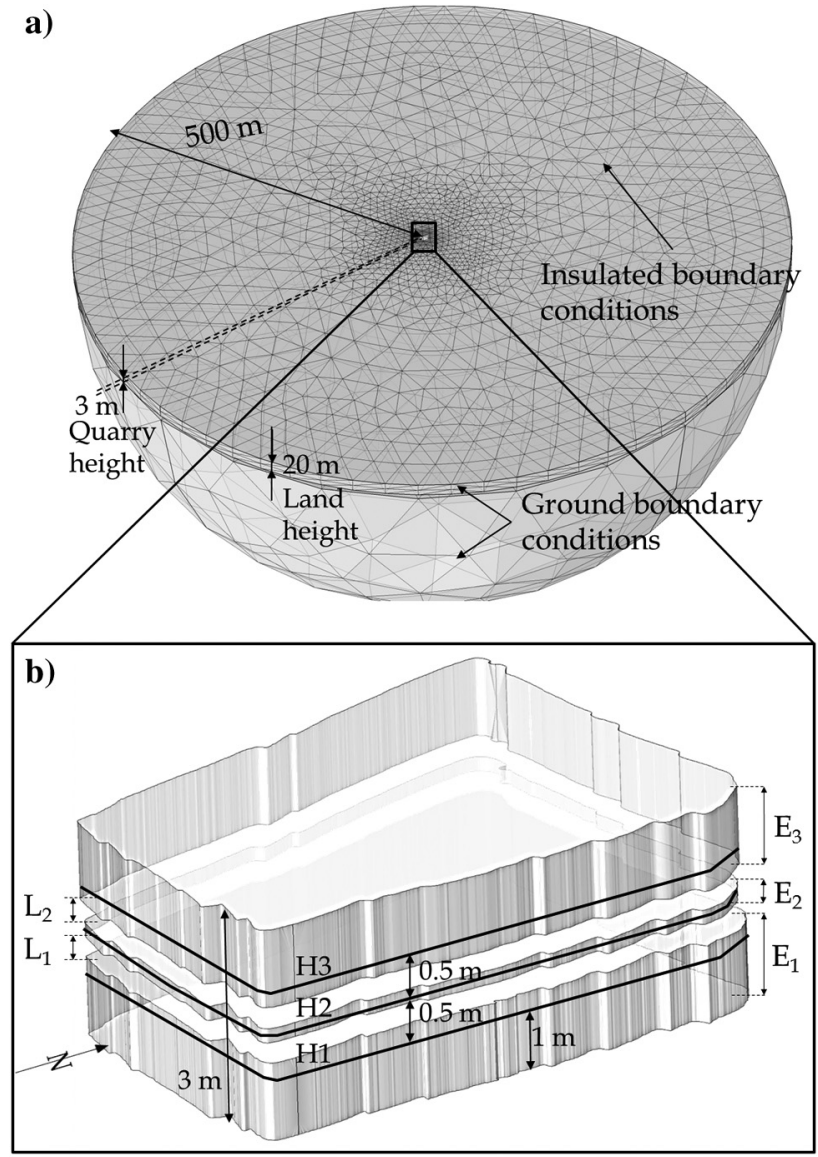

c)

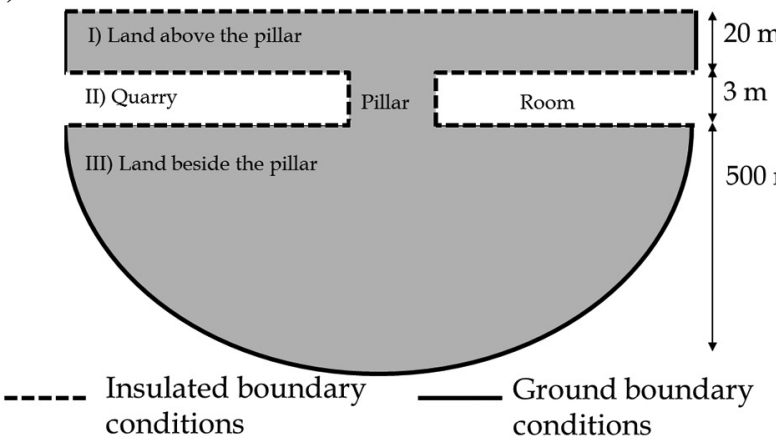

Figure 5. Description of the model with (a) mesh of the global model, (b) the central part of the model simulating the pillar, and (c) a simplified scheme section of the model; $E_{1}, E_{2}$, and $E_{3}$ represent extrusion zones correlated with the electrode belts; $\mathrm{L}_{1}$ and $\mathrm{L}_{2}$ show the loft zones in between the extrusion zones. The solid lines in $\mathrm{b}$ show the locations of the three electrode belts noted $\mathrm{H} 1, \mathrm{H} 2$, and $\mathrm{H} 3$. 
positions ( $\mathrm{H} 1, \mathrm{H} 2$, and $\mathrm{H} 3$ ). For $\mathrm{M} 1$, the three anomalies are clearly visible at the right location in the model for the three slices even if the model resolution decreases as distance from electrode increases (Rucker, 2012). The resistivity of A1 and A3 slightly differs from the true value, whereas the $A 2$ resistivity value is close to $150 \Omega \mathrm{m}$. However, because of the $\ell_{2}$-norm used, the inversion does not preserve the sharp interfaces between the anomalies and the pillar. Some small artifacts appear in the result in the vicinity of strong
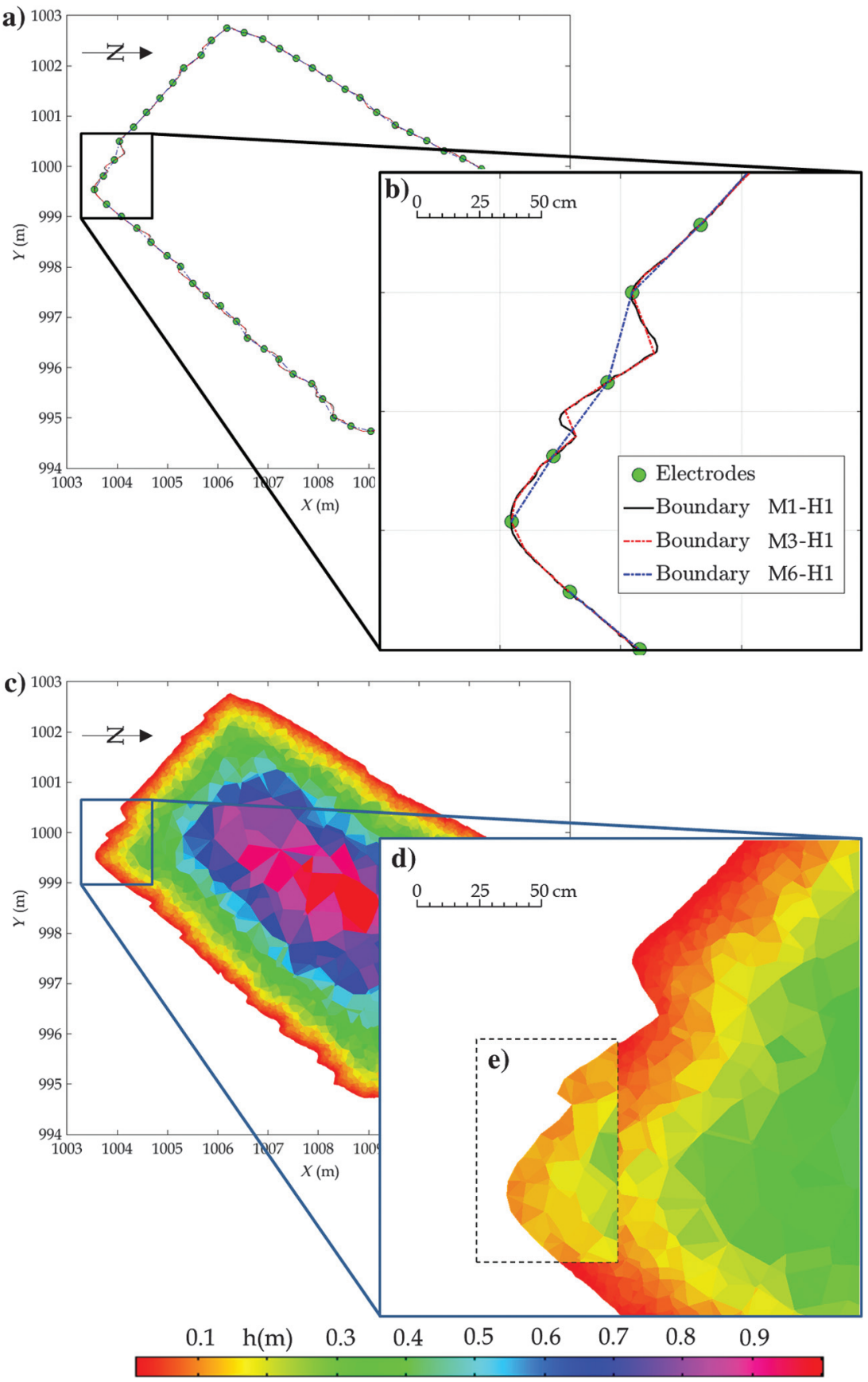

Figure 6. Sketch showing (a) various contours at height $\mathrm{H} 1$ used for the models M1, M3, and M6 and (b) an enlargement near a particular zone of M1 at height H1. The green points represent the locations of the electrodes. (c) A slice of the 3D forward mesh at H1, (d) enlargement of a zone of complex geometry, and (e) a slice of the 3D inversion cells. The color scale represents the average height $h$ of the meshes. working faces (near anomaly A1) and near anomaly A3. Because of the inherent loss of sensitivity in the center of the pillar, the detection capability of A3, as suggested by Tejero-Andrade et al. (2015), should be lower. In this study, we assume that the favorable context of our synthetic model facilitates the detection of A3. As proposed by Tejero-Andrade et al. (2015), optimized acquisition arrays could have been used to limit the loss of sensitivity and more accurately detect the central anomaly.

The model M2-M5 results are very similar to the previous model showing a high-quality reconstruction of the true model. The main visible difference between M1 and M2-M5 is the new artifacts in $\mathrm{H} 1, \mathrm{H} 2$, and $\mathrm{H} 3$ at various locations. The number of artifacts increases from M2 to M5. However, these artifacts do not limit detection of the three anomalies.

The result of M6 is different and presents various artifacts on the slices boundaries. The detection of the anomaly A1 is not possible, whereas the locations of anomalies A2 and A3 remain clear. Table 1 summarizes the input data of each study and information on the inversion results. The rms error increases from M1 to M6 showing the benefits of a more accurate surface geometry.

To enrich the comparison of the six cases investigated, the similarity index $S_{i}$ between the inversion result and the true model is used (equation 4). This index is computed for four different volumes: $S_{\mathrm{A} 1}, S_{\mathrm{A} 2}, S_{\mathrm{A} 3}$, and $S_{\mathrm{P}}$ corresponding to anomalies A1, A2, A3, and the embedding pillar body $\mathrm{P}$, respectively. Each index is computed on volumes whose height is bounded by the height of the pillar $(3 \mathrm{~m})$. All the similarity index values show a reconstruction error lower than $35 \%$ and show a decreasing quality from M1 to M6. This is particularly true for $S_{\mathrm{A} 1}$ and $S_{\mathrm{P}}$, indicating that a poor geometry description greatly affects the inversion results near the contour and in the vicinity of strong surface variations.

Concerning anomaly $\mathrm{A} 2$, the $S_{\mathrm{A} 2}$ results tend to show that the degradation of the geometry does not affect the inversion result far from the contour (at the heart of the pillar body). One unexpected result shows that the anomaly is better reconstructed in M5 compared with M3. This result may be due to a better model parameter discretization at the center of the pillar for M3. This difference of discretization can be explained by fewer model parameters near the electrodes for a quite constant number of model parameters in the six models.

The study of the similarity index of anomaly A3 shows little variation between the three models. However, an unexpected greater value is calculated for M1 than for M2. For all the unexpected results, this could be a side effect of the previously mentioned issue, or numerical issues, 
or more simply an over-interpretation of small variations in this criterion

Figure 9 shows the 3D inversion results (slice at $\mathrm{H} 1$ ) for three different noise levels $(1 \%, 5 \%$, and 10\%) and three surface geometry accuracies (M1, M3, and M6). We add a white Gaussian noise to "measured apparent resistivities" with a variance of one. The signal-to-noise ratio is adjusted to obtain the desired rms error $(1 \%$, $5 \%$, or $10 \%$ ). Results show that the anomaly A2 can be detected in all the results except Figure 9e and 9i; A1 is only detectable in the results of Figure 9a and A3 in the results of Figure 9a, 9b, and 9d. Results show that surface geometry accuracy could be compared with noise added to the measurements with small electrodes spacing. The second interpretation is that a higher noise level tends to smooth the reconstruction of A2, thus limiting its detectability. This study shows that a noise level superior to $5 \%$ and a few intermediate points inferior to five limits the resolution of the inversion result and its interpretability.

\section{Real case study}

This section focuses on the data measured on the real underground mine pillar performed in June 2015 and presented in the section "Site test description." The most complete model (M1) of the previous section is used because it supplies the best choice in terms of results and computing cost. In this study, M6 is also used with the same data set to help identify artifacts due to a poor surface geometry description.

Figure 10 shows for M6 (left column) and M1 (right column) three slices representing the electric resistivity distribution extracted from the 3D inverse model at heights $\mathrm{H} 1, \mathrm{H} 2$, and $\mathrm{H} 3$. The slices are extracted at iteration 4 to limit the over-fitting phenomenon and

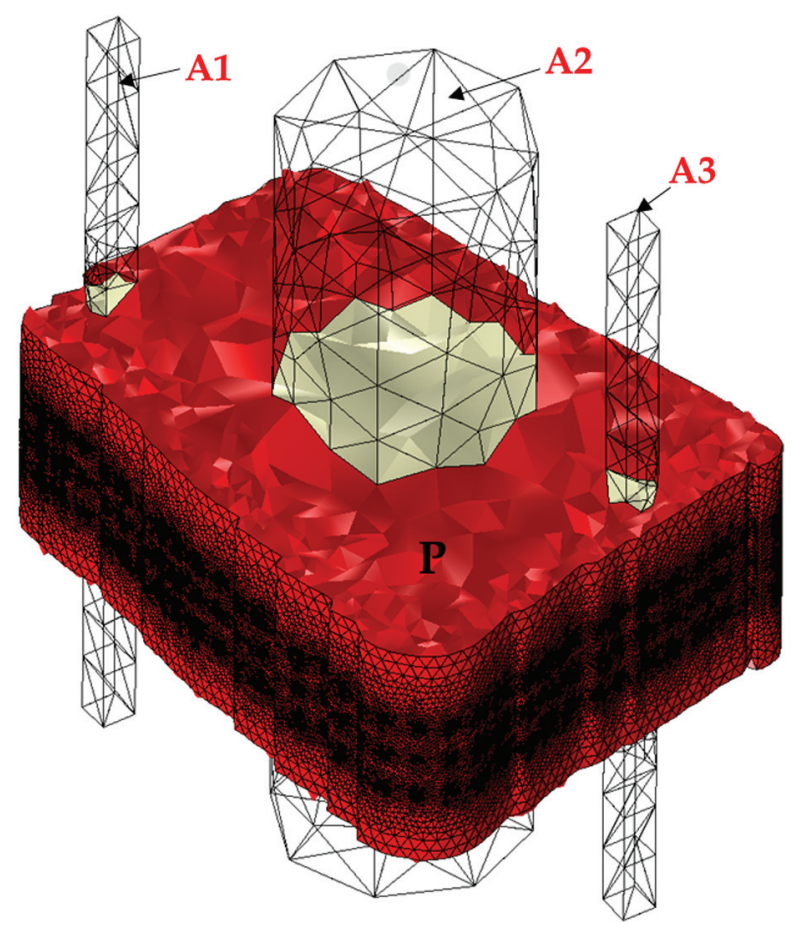

Figure 7. 3D view of the center part (the pillar) of the true model mesh with anomalies A1, A2, and A3. The beige and red colors refer to resistivity of 150 and $250 \Omega \mathrm{m}$, respectively. present an rms error of $6.78 \%$ and $5.25 \%$ for M6 and M1, respectively. In the following, M6 inversion results will only be discussed to help the interpretation of the M1 inversion results. For the latter, the distribution is quite homogeneous with a mean resistivity of $250 \Omega \mathrm{m}$, but it presents a few areas where the resistivity ranges from 150 to $350 \Omega \mathrm{m}$. Overall, the three slices not only present a similarity due to a small distance between the belts but also some variations (resistive and conductive) in some particular zones of interest. Some highly resistive zones are persistent on two slices $\left(\mathrm{Ar}_{1}\right.$, $\mathrm{Ar}_{3}$, and $\mathrm{Ar}_{4}$ ) but will be considered as artifacts due to the presence of an over-structuration phenomenon (a resistive zone neighboring a conductive zone) or due to strong surface geometry variations in the vicinity. Other highly resistive zones are considered as artifacts due to (1) their nonpersistence on other slices, (2) the presence of an

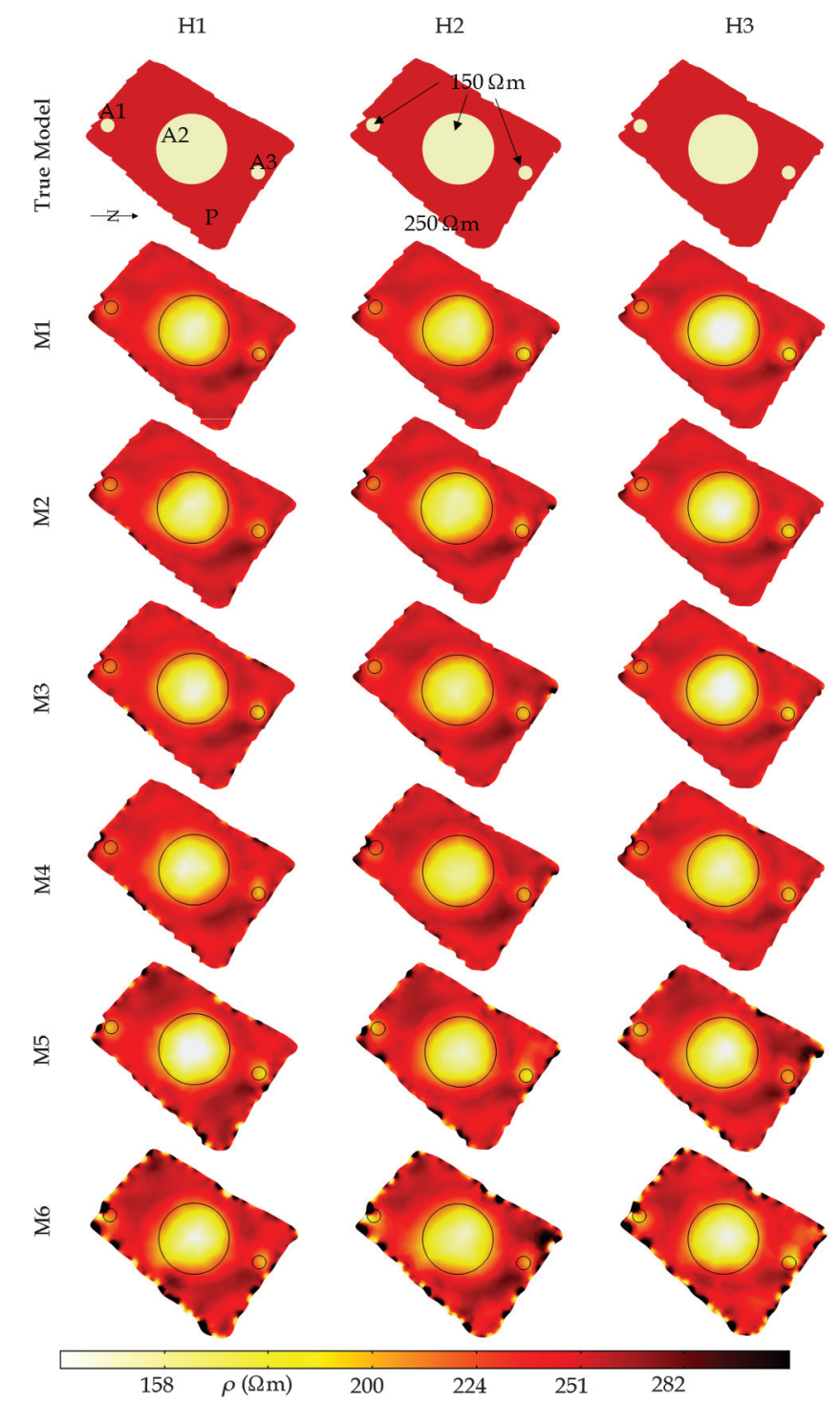

Figure 8 . The synthetic study results, each column corresponding to a slice of a 3D model at height $\mathrm{H} 1, \mathrm{H} 2$, and $\mathrm{H} 3$. The first line shows the true model used for the synthetic study. Rows two to seven show the inversion results corresponding to cases M1-M6, respectively. The locations of the anomalies (A1, A2, and A3) are superimposed on the inversion results (circles). 
over-structuration phenomenon, and (3) strong variations in the surface section geometry nearby $\left(\mathrm{Ar}_{2}, \mathrm{Ar}_{5}, \mathrm{Ar}_{6}, \mathrm{Ar}_{7}, \mathrm{Ar}_{11}\right.$, and $\left.\mathrm{Ar}_{12}\right)$. Two conductive zones are considered as artifacts $\left(\mathrm{Ar}_{9}\right.$ and $\mathrm{Ar}_{10}$, see $\mathrm{H} 2$ ) due to a small persistence on the other slices and the presence of important surface geometry variations nearby.

A fast zoning is presented on each slice to delineate conductive or resistive zones. Four clusters are defined: (1) a conductive "C," (2) a moderately conductive "MC," (3) a moderately resistive "MR," and (4) a resistive cluster "R." This zoning shows that the medium is quite homogeneous beyond $50 \mathrm{~cm}$ inside the pillar.

Each slice shows persistent resistive and conductive zones that are essential for the risk assessment interpretation. Indeed, Gombert et al. (2013) show that increasing the water content decreases the strength resistance of chalk. Figure 11 summarizes the main results

Table 1. Information about both entries of the six models and inversion results.

\begin{tabular}{lcccccc}
\hline & M1 & M2 & M3 & M4 & M5 & M6 \\
\hline Number of intermediate points & $\sim 40$ & 10 & 5 & 2 & 1 & 0 \\
Distance interpoints (cm) & 1 & 4 & 7 & 13 & 20 & 40 \\
Number of inversion parameters & 22,788 & 23,817 & 22,873 & 22,646 & 22,663 & 22,438 \\
The rms error at iteration 7 (\%) & 0.009 & 0.013 & 0.036 & 0.073 & 0.136 & 0.412 \\
$S_{\mathrm{A} 1}$ & 0.188 & 0.212 & 0.224 & 0.235 & 0.260 & 0.354 \\
$S_{\mathrm{A} 2}$ & 0.12 & 0.122 & 0.134 & 0.134 & 0.132 & 0.162 \\
$S_{\mathrm{A} 3}$ & 0.124 & 0.133 & 0.126 & 0.137 & 0.148 & 0.162 \\
$S_{\mathrm{P}}$ & 0.089 & 0.096 & 0.105 & 0.125 & 0.210 & 0.324 \\
\hline
\end{tabular}

of this study with the photogrammetric model and the 3D inversion result (extracted slices). This representation provides better understanding of the pillar and its electric properties. It also leads to an improved interpretation of the available data for a better assessment of the structure. The observed resistivity variations are consistent with the results of Lafrance et al. (2016) on two pillars of different lithotypes. In their study, some laboratory tests on core samplings are done from the wall to the center of the pillars. Among all results, the connected porosity varies between $25 \%$ and $50 \%$. Following Archie's law, this porosity variation can induce a resistivity variation of 1:5 (for a saturated soil medium and a cementation exponent of two). This result is consistent with the global resistivity variation inside the pillar (150-400 $\Omega \mathrm{m}$ ). In Figure $11 \mathrm{~b}$, we propose a simplified interpretation of the inversion results. Two phenomena seem to be at the origin of the resistivity variations.

First, the geologic variability of the chalk during the sedimentation with an irregular distribution of macroflints and fossils. Second, a vertical drainage through the pillar takes place as proposed by Lafrance et al. (2016). Lafrance et al. (2016) suggest that an increase in connected porosity is therefore a direct effect of dissolution. The drainage plays a key role in the erosion and dissolution process via chemical weathering for the latter. The drainage in the pillar is amplified by two factors: first, the "room and pillar" organization of the quarry increasing water circulation inside the pillar and, second, the presence of cracks due to a collinear fault family. This phenomenon that is visible on the pillar (Figure 11c) can increase microcracks of the chalk and so the porosity. This conclusion is consistent with the results of Lafrance et al. (2016) who propose that the amount "of cracks and pores should be greater at the pillar walls than in the middle of the pillar."

\section{DISCUSSION}

On the one hand, the 3D media of complex geometry such as underground mine pillars cannot be assessed by conventional geoelectric imaging tools. On the other hand, the photogrammetric technique is increasingly used to provide an accurate cost-effective 3D surface description. This paper shows that the sequential combination of photogrammetric and ERI methods proves particularly relevant for the assessment of media of complex 3D geometry. The second finding is that the accuracy of the geometry (or DTM) is correlated with the inversion reliability.

This research follows the works of Sasaki (1994) and more recently the papers of Demirci et al. (2012) and Erdoğan et al., 2008 introducing the problem of topography in the inversion process. The latter present five different solutions based on finite-difference or finite-element modeling and with a half or full representation of the air volume. In contrast with their paper, we choose to explicitly mesh a $3 \mathrm{D}$ complex volume without applying any mesh deformation procedure. This difference adds a step to the inversion process but overcomes the error associated with mesh deformation.

This paper also contrasts with the conventional technique used to obtain the surface model. We present the first attempt to explicitly take into account an output photogrammetric model as an ERI input model geometry. For this particular point in our approach, the
Figure 9. The combined effect of noise (levels: $1 \%, 5 \%$, and $10 \%$ ) and surface geometry accuracy (models: M1, M3, M6) on inversion results. The sections are extracted at height $\mathrm{H} 1$ of the $3 \mathrm{D}$ inversion model at iteration 7 . 
choice of the photogrammetric method instead of laser technology leads to a cost-effective survey solution. As explained by Bretar et al. (2013), the result of the photogrammetric stage is a surface point cloud exhibiting a resolution better than $2 \mathrm{~cm}$. Results show that it also offers a greater flexibility to obtain an optimized surface reconstruction near areas of interest (cutting face, electrodes). However, the application to an underground mine requires the scene to be lightened.

This paper in one particular aspect goes further than the research presented by Erdoğan et al. (2008), which takes into account only the electrode locations to create the model. We demonstrate here the need to use topography information between electrodes and in their vicinity. Consequently, this result ensures consistency with Maurer and Friedel (2006), who show that it is necessary to integrate information on the medium even in regions away from the electrodes. Moreover, it brings clues as to the resolution needed in between electrodes. For complex models, five elevation points in between each electrode seem to be a minimum trade-off between the inversion quality and the cost of the photogrammetric survey. Conversely, it shows that just a perfect knowledge of the location of the electrodes

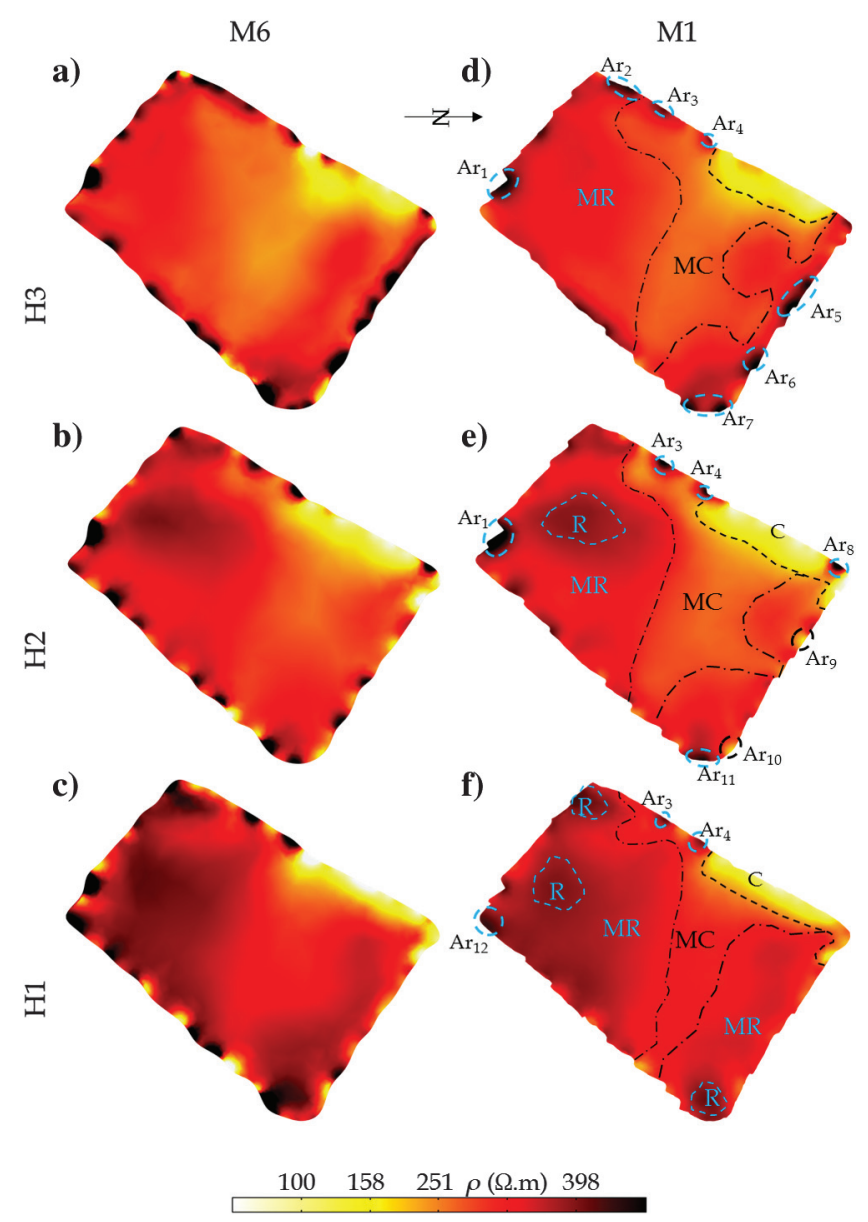

Figure 10. The block figure presenting pillar results of (a-c) model M6 and (d-f) model M1. Each column corresponds to a slice of a 3D model (iteration 5) at heights $\mathrm{H} 1, \mathrm{H} 2$, and $\mathrm{H} 3$. The location of the artifacts (Ari) and zoning are superimposed on the inversion results ( $\mathrm{R}$, resistive; MR, moderately resistive; $\mathrm{MC}$, moderately conductive; and $\mathrm{C}$, conductive). (without other geometric information) is not sufficient to achieve a relevant inversion result.

This procedure was applied to a real quarry pillar with a resolution on the model geometry never taken into account before. Due to numerical issues, the complete geometry was not taken into account, leaving questions about the presence of residual artifacts in the inversion result. Due to the construction of the geometry with horizontal slices, the questioning more particularly concerns
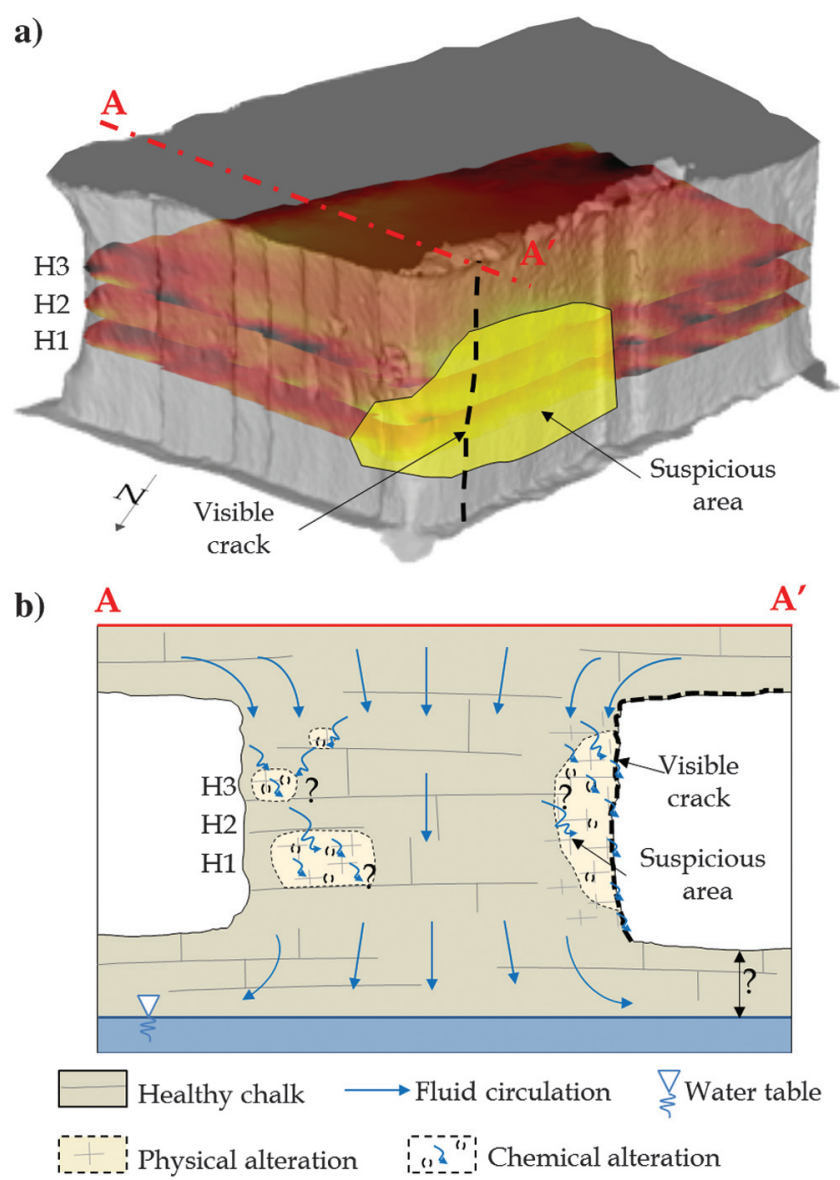

c)

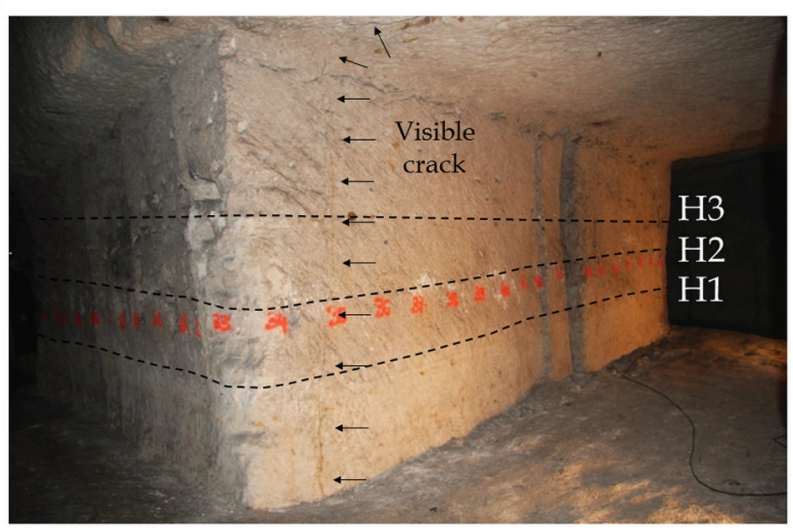

Figure 11. The synthesis of the results with (a) a 3D view of the result of the photogrammetric and ERI surveys, (b) a geologic interpretation of the results, and (c) a picture of the pillar. Some additional information is superimposed such as the location of a visible crack on the surface and the extension of the "suspicious area." 
an incomplete vertical resolution. The extrusion and loft operations greatly limit vertical variations that can occur in the vicinity of electrodes. This result confirms the conclusions of Sjödahl et al. (2006) showing that the lateral resistivity variations have an important effect on the measurement itself. This incomplete surface reconstruction appears as one of the main causes of artifact generation in the inversion process. As demonstrated by the synthetic study, the noise on measurement can also generate artifacts and limits the detectability of anomalies inside the pillar. However, main artifacts appear near great surface topography variations.

Results in this paper imply that for the common practice of performing a DTM, one has to take particular care during a geophysical survey. For the example of volcano surveys (Finizola et al., 2002; Brothelande et al., 2014, 2015), or engineering applications (hydraulic structures [Fargier et al., 2014]), the desired ERI resolution is often weaker than the DTM variations between two adjacent electrodes. In this case, our experience shows that the use of a very accurate surface description must become of major importance when the variation between interelectrode spacing and surface electrode spacing (at the same scale) is greater than $30 \%$.

\section{CONCLUSION}

A methodology of sequentially combining photogrammetry and ERI is presented and applied to an underground mine pillar to fully process a considerable amount of data. The photogrammetric technique provides a low-cost, high-resolution surface model, set as input geometry data for the ERI inversion. A new ERI inversion code based on MATLAB and Comsol Multiphysics software was developed to fully invert the $3 \mathrm{D}$ model. This paper presents the first attempt to fully integrate an output photogrammetric model as an input model for ERI. The paper focuses on one particularly difficult issue of the methodology that consists in adapting the output photogrammetric model to the inversion code. An empirical procedure based on postprocessing stages is proposed to supply a surface model suitable for the 3D ERI. This step is followed by a numerical test showing the negative effect of an incomplete resolution of the photogrammetric model used as an input model for ERI. Some recommendations can be derived from results concerning a minimum acceptable resolution of the photogrammetric model. The methodology is applied to a real case study of an underground mine pillar of chalk. Despite the complex geometry of the examined pillar, our methodology provides reliable 3D inversion results. However, some artifacts partly due to an incomplete vertical resolution of the input model geometry remain present but can be identified by superimposing the ERI reconstruction and the photogrammetric result. In the near future, we hope first to improve this particular point in order to better take vertical variations between electrode belts into account. Further research will then focus on an experimental procedure to link electric resistivity spatial evolution, water content, and unconfined compressive strength to supply a safety criterion for the pillar.

\section{ACKNOWLEDGMENTS}

The authors wish to thank the city council of Vendôme for access to the site as well as the research projects PRECAS and APHOGEOPHY for funding. We are grateful to E. Pairault, Y. Boussafir, and J. Lacogne for field data collection and interpretation.

\section{REFERENCES}

Abellán, A., T. Oppikofer, M. Jaboyedoff, N. J. Rosser, M. Lim, and M. J. Lato, 2014, Terrestrial laser scanning of rock slope instabilities: Earth Surface Processes and Landforms, 39, 80-97, doi: 10.1002/esp.v39.1.

Al Heib, M., C. Duval, F. Theoleyre, J.-M. Watelet, and P. Gombert, 2014, Analysis of the historical collapse of an abandoned underground chalk mine in 1961 in Clamart (Paris, France): Bulletin of Engineering Geology and the Environment, 74, 1001-1018, doi: 10.1007/s10064-014-0677-6.

Audebert, M., R. Clément, J. Grossin-Debattista, T. Gunther, N. TouzeFoltz, and S. Moreau, 2014, Influence of the geomembrane on time-lapse ERT measurement for leachate injection monitoring: Waste Management, 34, 780-790, doi: 10.1016/j.wasman.2014.01.011.

Bell, F. G., M. G. Culshaw, B. S. P. Moorlock, and J. C. Cripps, 1992, Subsidence and ground movements in chalk: Bulletin of the International Association of Engineering Geology, 45, 75-82, doi: 10.1007/BF02594906.

Brady, B. H. G., and E. T. Brown, 2013, Rock mechanics for underground mining: Springer Science \& Business Media.

Bretar, F., M. Arab-Sedze, J. Champion, M. Pierrot-Deseilligny, E. Heggy, and S. Jacquemoud, 2013, An advanced photogrammetric method to measure surface roughness: Application to volcanic terrains in the Piton de la Fournaise, Reunion Island: Remote Sensing of Environment, 135, 1-11, doi: 10.1016/j.rse.2013.03.026.

Brothelande, E., A. Finizola, A. Peltier, E. Delcher, J.-C. Komorowski, F. Di Gangi, G. Borgogno, M. Passarella, C. Trovato, and Y. Legendre, 2014, Fluid circulation pattern inside La Soufrière volcano (Guadeloupe) inferred from combined electrical resistivity tomography, self-potential, soil temperature and diffuse degassing measurements: Journal of Volcanology and Geothermal Research, 288, 105-122, doi: 10.1016/j.jvolgeores.2014.10.007.

Brothelande, E., J.-F. Lénat, A. Normier, C. Bacri, A. Peltier, R. Paris, K. Kelfoun, O. Merle, A. Finizola, and E. Garaebiti, 2015, Insights into the evolution of the Yenkahe resurgent dome (Siwi caldera, Tanna Island, Vanuatu) inferred from aerial high-resolution photogrammetry: Journal of Volcanology and Geothermal Research, 299, 78, doi: 10.1016/j .jvolgeores.2015.04.006.

Brunet, P., R. Clément, and C. Bouvier, 2010, Monitoring soil water content and deficit using electrical resistivity tomography (ERT): A case study in the Cevennes area, France: Journal of Hydrology, 380, 146-153, doi: 10 $.1016 /$ j.jhydrol.2009.10.032.

Chandler, J. H., J. G. Fryer, and A. Jack, 2005, Metric capabilities of lowcost digital cameras for close range surface measurement: The Photogrammetric Record, 20, 12-26, doi: 10.1111/j.1477-9730.2005.00302.x.

Cho, I. K., and J. Y. Yeom, 2007, Crossline resistivity tomography for the delineation of anomalous seepage pathways in an embankment dam: Geophysics, 72, no. 2, G31-G38, doi: 10.1190/1.2435200.

Dahlin, T., 1996, 2D resistivity surveying for environmental and engineering applications: First Break, 14, 275-283, doi: 10.3997/1365-2397.1996014.

deGroot-Hedlin, C., and S. Constable, 1990, Occam's inversion to generate smooth, two-dimensional models from magnetotelluric data: Geophysics, 55, 1613-1624, doi: 10.1190/1.1442813.

Demirci, I., E. Erdoğan, and M. E. Candansayar, 2012, Two-dimensional inversion of direct current resistivity data incorporating topography by using finite difference techniques with triangle cells: Investigation of Kera fault zone in western Crete: Geophysics, 77, no. 1, E67-E75, doi: 10 $.1190 /$ geo2011-0130.1.

Dérobert, X., and O. Abraham, 2000, GPR and seismic imaging in a gypsum quarry: Journal of Applied Geophysics, 45, 157-169, doi: 10.1016/ S0926-9851(00)00025-2.

Erdoğan, E., I. Demirci, and M. E. Candansayar, 2008, Incorporating topography into 2D resistivity modeling using finite-element and finite-difference approaches: Geophysics, 73, no. 3, F135-F142, doi: 10.1190/1.2905835.

Fargier, Y., S. Palma Lopes, C. Fauchard, D. François, and P. Côte, 2014, DC-Electrical resistivity imaging for embankment dike investigation: A 3D extended normalisation approach: Journal of Applied Geophysics, 103, 245-256, doi: 10.1016/j.jappgeo.2014.02.007.

Fauchard, C., R. Antoine, F. Bretar, J. Lacogne, Y. Fargier, C. Maisonnave, V. Guilbert, P. Marjerie, P.-F. Thérain, J.-P. Dupont, and M. Pierrot-Deseilligny, 2013, Assessment of an ancient bridge combining geophysical and advanced photogrammetric methods: Application to the Pont De Coq, France: Journal of Applied Geophysics, 98, 100-112, doi: 10.1016/j .jappgeo.2013.08.009.

Ferrero, A. M., A. Segalini, and G. P. Giani, 2010, Stability analysis of historic underground quarries: Computers and Geotechnics, 37, 476-486, doi: 10.1016/j.compgeo.2010.01.007.

Finizola, A., F. Sortino, J.-F. Lénat, and M. Valenza, 2002, Fluid circulation at Stromboli volcano (Aeolian Islands, Italy) from self-potential and $\mathrm{CO}_{2}$ surveys: Journal of Volcanology and Geothermal Research, 116, 1-18, doi: 10.1016/S0377-0273(01)00327-4.

Fox, R. C., G. W. Hohmann, T. J. Killpack, and L. Rijo, 1980, Topographic effects in resistivity and induced-polarization surveys: Geophysics, 45, 75-93, doi: 10.1190/1.1441041. 
Gombert, P. C. Auvray, and M. A. Heib, 2013, In-situ and laboratory tests to evaluate the impact of water table fluctuations on stability of underground chalk mines: Procedia Earth and Planetary Science, 7, 304-308, doi: 10 .1016/j.proeps.2013.03.138.

Günther, T., C. Rücker, and K. Spitzer, 2006, Three-dimensional modelling and inversion of DC resistivity data incorporating topography - Part 2: Inversion: Geophysical Journal International, 166, 506-517, doi: 10 $.1111 / \mathrm{j} .1365-246 \mathrm{X} .2006 .03010 . x$.

Hayley, K., L. Bentley, and A. Pidlisecky, 2010, Compensating for temperature variations in time-lapse electrical resistivity difference imaging: Geophysics, 75, no. 4, WA51-WA59, doi: 10.1190/1.3478208.

Jones, G., P. Sentenac, and M. Zielinski, 2014, Desiccation cracking detection using 2-D and 3-D electrical resistivity tomography: Validation on flood embankment: Journal of Applied Geophysics, 106, 196-211, doi: 10.1016/j.jappgeo.2014.04.018.

Lafrance, N., C. Auvray, M. Souley, and V. Labiouse, 2016, Impact of weathering on macro-mechanical properties of chalk: Local pillar-scale study of two underground quarries in the Paris Basin: Engineering Geology, 213, 107-119, doi: 10.1016/j.enggeo.2016.08.014.

Lesparre, N., B. Grychtol, D. Gibert, J.-C. Komorowski, and A. Adler, 2014 Cross-section electrical resistance tomography of La Soufrière of Guadeloupe lava dome: Geophysical Journal International, 197, 1516-1526, doi: $10.1093 /$ gji/ggu104.

Li, S., B. Liu, L. Nie, Z. Liu, M. Tian, S. Wang, M. Su, and Q. Guo, 2015, Detecting and monitoring of water inrush in tunnels and coal mines using direct current resistivity method: A review: Journal of Rock Mechanics and Geotechnical Engineering, 7, 469-478, doi: 10.1016/j.jrmge.2015 .06 .004 .

Loke, M. H., and R. D. Barker, 1996, Rapid least-squares inversion of apparent resistivity pseudosections by a quasi-Newton method: Geophysical Prospecting, 44, 131-152, doi: 10.1111/j.1365-2478.1996.tb00142.x.

Marescot, L., S. Palma Lopes, S. Rigobert, and A. G. Green, 2008, Nonlinear inversion of geoelectric data acquired across 3D objects using a finite-element approach: Geophysics, 73, no. 3, F121-F133, doi: 10 $.1190 / 1.2903836$.
Maurer, H., and S. Friedel, 2006, Outer-space sensitivities in geoelectrical tomography: Geophysics, 71, no. 3, G93-G96, doi: 10.1190/1.2194891. Multiphysics C, 2008, AC/DC module: User's guide: Comsol.

Oldenborger, G. A., P. S. Routh, and M. D. Knoll, 2005, Sensitivity of electrical resistivity tomography data to electrode position errors: Geophysical Journal International, 163, 1-9, doi: 10.1111/j.1365-246X.2005.02714.x.

Park, S. K., and G. P. Van, 1991, Inversion of pole-pole data for 3-D resistivity structure beneath arrays of electrodes: Geophysics, 56, 951-960, doi: $10.1190 / 1.1443128$

Pierrot-Deseilligny, M., and I. Clery, 2011, APERO: An open source bundle adjustment software for automatic calibration and orientation of set of images: International Archives of Photogrammetry, Remote Sensing and Spatial Information Sciences, 38, 5W16.

Pierrot-Deseilligny, M., and I. Clery, 2012, Some possible protocols of acquisition for optimal use of the "APERO" open source software in automatic orientation and calibration: EuroCOW Workshop.

Rings, J., A. Scheuermann, K. Preko, and C. Hauck, 2008, Soil water content monitoring on a dike model using electrical resistivity tomography: Near Surface Geophysics, 6, 123-132, doi: 10.3997/1873-0604.2007038.

Rucker, D. F., 2012, Enhanced resolution for long electrode ERT: Geophysical Journal International, 191, 101-111, doi: 10.1111/j.1365-246X.2012 .05643.X.

Sasaki, Y., 1994, 3-D resistivity inversion using the finite-element method: Geophysics, 59, 1839-1848, doi: 10.1190/1.1443571.

Sjödahl, P., T. Dahlin, and B. Zhou, 2006, 2.5D resistivity modeling of embankment dams to assess influence from geometry and material properties: Geophysics, 71, no. 3, G107-G114, doi: 10.1190/1.2198217.

Tejero-Andrade, A., G. Cifuentes, R. E. Chavez, A. E. Lopes-Gonzales, and C. Delgado-Solorzano, 2015, L- and CORNER-arrays for 3D electric resistivity tomography: An alternative for geophysical surveys in urban zones: Near Surface Geophysics, 13, 355-367, doi: 10.3997/1873-0604.2015015.

Vedaldi, A., and B. Fulkerson, 2010, VLFeat: An open and portable library of computer vision algorithms: Proceedings of the 18th ACM International Conference on Multimedia, ACM, 1469-1472, doi: 10.1145/ 1873951.1874249 . 